\title{
Semantic Web for Program Administration
}

\author{
doi:10.3991/ijet.v5i4.1434 \\ Q. A. Memon ${ }^{1}$, S. A. Khoja ${ }^{2}$ \\ ${ }^{1}$ UAE University, Al Ain, United Arab Emirates \\ ${ }^{2}$ IBA, Karachi, Pakistan
}

\begin{abstract}
Typically, administrative systems involving information in an academic environment are disjoint, spread across various departments and support independent queries. The objectives in this work include investigation on integrating these independent systems to provide support to intelligent queries run on the integrated platform. A framework is proposed that enriches data in the legacy systems to provide a value-added semantic layer where annotation, query and reasoning can be carried out to support management requirements. The development of this framework with a case study of a typical engineering program is discussed to show how semantic web technologies can be used by stack holders of the program for better academic program administration. The comparative work is described to show applications where semantic web have been explored. The issues related to implementation of the semantic web to academic program administration are also highlighted and discussed.
\end{abstract}

Index Terms-Program Administration, Semantic Web, Web Technology, Integrated Academic Systems

\section{INTRODUCTION}

Unlike Content Management Systems (CMS) [1-2] that provide educational services, such as Virtual Learning Environments (VLEs), course repositories, library archives, online examinations, online coursework submission, etc., the learning process management systems use Web-based technology to plan, implement, and assess a specific learning process. This technology allows the employee/student to take learning into their own hands while either staying current in their specific field or branch out and learning new skills. On the other hand, Academic Information and Management Systems (AIMS) [3-4] are mostly used in academic environment to support information, finance, logistics, human resource and student services. Both types of systems create huge databases containing interrelated data. Generally, the academic and content management systems work in isolation (mostly maintained by different departments) and in many cases, not even designed to interact with each other at later stages [2].

The universities, in general use such sub-systems, most of them independent to carry out specific tasks in an academic administration environment. In order to exemplify this, example sub-systems along with respective functionalities (typically found at UAE University [5]) are:

a) Blackboard system: contains course logs, student assessment data done by faculty, course evaluation done by students - users are students, faculty, and administration.

b) Enterprise Administrative System: Enterprise Administrative System also sometimes referred to as
Banner System is a system used by a number of administrative departments to maintain employee \& student information. It contains student, finance, and human resource (HR)/Payroll information - users are administration and University Registrar.

c) Electronic services system: contains employee information (personal data, time sheets, benefits, job data, paystubs), entering grades by faculty, registration overrides, view class lists and student information, HR system - users are faculty, student advisors, staff, administration.

d) Faculty evaluation system: contains faculty members' annual evaluation reports - users are faculty, administration.

e) Research Affairs system: contains data related to research grants related to faculty, local conferences users are faculty, research affairs management, and public.

f) Email server: contains email logs - users are administration, faculty, staff, students

g) Web server: contains websites of colleges (and departments), faculty web pages, data related to industrial training of students, graduation projects of students - users are administration, faculty, students, and public.

h) College server: Each college of the university has its own server containing logs about programs, courses, accreditation body, program assessments, etc.

This list varies from institution to institution. The subsystems individually yield long and repetitive work to reach a level of decision making related to education administration. The individual systems do not open up the data for collaborative reuse of the learning resources to help reason a higher level intelligent query. Though, some of the universities have exercised efforts to create a data level bridge between various packages, but no formal integration or data association has been implemented to infer linked information other than the data itself.

The growth of an academic system is measured through its evolution. Typically, the stake holders include students, faculty, administration, local industry and professional bodies etc. In absence of collaborative subsystems, the decision making in a distributed system would require tiring analysis of extensive data resulting in evolving rate that may not keep up to local industry needs. The yield from an academic system relies heavily on timely collection of data from stake holders and decision making based on faculty-administration nexus. With reference to engineering education, this slows down the local and international accreditation efforts.

Generally, the objective set behind deployment of academic systems is integration of organizational processes that are distributed but not formally designed for interac- 
tion amongst its distributed entities. As an example, a technological system such as Enterprise Resource Planning (ERP) integrates the data and processes of an organization into one single system. Such ERP systems, for example systems application product (SAP), People soft, etc. work on fixed queries and return atomic results, whereas semantic web returns quasi queries. The semantic web [6] is a web of machine processable meanings underpinned by shared and formally defined ontologies. According to Wikipedia [7], the Semantic Web is an evolving development of the World Wide Web in which the meaning (semantics) of information and services on the web is defined, making it possible for the web to understand and satisfy the requests of people and machines to use the web content. It derives from World Wide Web Consortium director Sir Tim Berners-Lee's vision of the Web as a universal medium for data, information, and knowledge exchange [8]. For ERPs, the databases have to be properly normalized, whereas semantic works on any relation which has been defined through ontologies. Semantic web technologies aim to open up the data by providing more flexible ability of collaborative annotation and reuse of the learning resources.

Ontology is a formal representation of a set of concepts within a domain and the relationships between those concepts. It is used to reason about the properties of that domain, and may be used to define the domain. In order to coordinate different semantic web activities, an educational ontology may be explicitly defined to share a contextual conceptualization of the educational domain, which can be then used to annotate lecture resources, program specifications, modules, assessments, etc. This allows the users to make their resources more machineprocessable by collaboratively constructing an enriched layer of the semantic web that links educational artefacts with formal semantics to support other semantic activities such as semantic query, aggregation and reasoning [9]. As semantic web opens up the data for reuse of learning resources within a domain (for example in an engineering college), the relations amongst data related to various college departments become clearer and thus rationale may easily be developed to initiate possible joint/multidisciplinary programs or identify common weaknesses within college departments.

In this paper, the context of engineering education is used to demonstrate the practice of semantic web activities such as semantic annotation, query and reasoning. In section 2, the sample queries related to a typical engineering program administration process at a university are briefly outlined to depict some operational instances, which may be used to develop respective ontology specification. Section 3 discusses in detail the development of a typical (UAE University-Engineering) ontology; how semantic annotations may be generated and reused with example queries that are used for implementation of the academic scenario. The section 4 discusses some of the important related works related to semantic web, followed by discussions in section 5 . The conclusions made during the implementation process are presented in section 6.

\section{RELATED WORK}

In an academic environment, the main outcomes of semantic web technologies are considered as information gathering, collaborative teaching and learning activities, such as team building, content creation and formation of well formed metadata for content. One of the implementation examples discussed in [10] enables students to access and search over a number of pre-selected and semantically matched to curriculum online resources that include books, multimedia and resources from encyclopaedias. These resources may be clustered as per students' age group and automatically 'pushed' to students whilst they work. Another example is a scheduling tool [11] to help students at Massachusetts Institute of Technology (MIT) to plan their course subjects using a semantic data source. This system uses well formed metadata of the university's official course catalogue, generating time tables, course loads, and folksonomy of students rating for a particular course. The objective of the work in [12] is to develop a prototype of a distributed network of semantically aware shared annotated services, in the form of RDF stores, resulting in a semantic layer to support a cluster of applications which will either directly support users in finding and recovering useful resources in a particular academic domain, or indirectly support students by supporting user-facing applications. The implementation example in [13] is a web based assessment engine in addition to computing statistics that are tied directly to program outcomes for accreditation process implementation. In another effort, Ed-Scene [14] is an effort to combine all the information and data available in an academic system in order to develop scenarios using semantic web to support different roles (such as teachers, students, quality assurance people, management, perspective employers, etc.) in an academic system.

In all the above examples it is clear that semantic technologies aspire to make substantial reuse of existing data and ontologies, shaping as linked information space in which data is being enriched and added. The targeted advantages noted during this study for semantic web applications were explicit student related services, management of a set of services or the assessment. In this paper, an entire academic environment is investigated to employ semantic layer at the highest layer to open the data in multiple domains for collaborative use. In turn, this may help certain aspects of education (for example engineering discipline) to evolve to the expectations of academic administration and industry. Thus, effective use of data in multiple domains may help identify interdisciplinary programs, bring uniformity in academic systems within a college/university, validate local and international accreditation process, measure industrial contribution to academics, etc.

\section{ACADEMIC PROGRAMME AdMinistration MANAGEMENT}

Generally, the integration of academic environment sub-systems is also meant for providing support to all stakeholders of an academic system for solving intelligent queries. Intelligent query is defined as searching and mining large knowledge bases which are collections of atomic facts and general rules (horn clauses), the rules should be allowed to occur in the answer for a query [15]. The following are some of the example engineering education scenarios that are being addressed in this research:

a) The department wishes to allocate a course, for which no straight matching to any lecturer is available. A layer can be developed to identify near 
matching of skills of lecturers to teach a course. Similarly, database of teaching load can be attached to check the loading of the teachers.

b) A group of students have completed core courses and are in final year for elective/specialization courses. The department wishes to know the size of students per each elective course with a requirement of grading point average (GPA) of 3.0 and above in design and simulations. The students belong to different programs: Electrical Engineering, Communications Engineering and Computer Engineering.

c) The department is interested in comparing two groups for strengths and skills in engineering design. Each group graduated recently from a different engineering program. The resulting data is to be provided to stakeholders for comparative purposes. The criterion that may be used includes typically the number of elective courses completed.

d) The college is interested in comparing performance of its students in science and mathematics in order to select a group of students to send it to a science exhibition. The students belong to various departments of the college.

e) The department is interested in comparative assessment of senior (final) year project taken by last three graduating batches. The assessment shall provide the number of students, project type (whether design and simulate or design and build), respective grades obtained, and the opinion of different employers in the country about graduates (as employees).

f) The department is interested in evaluating the performance of delivery of core courses in various programs offered in the department. For this, it intends to compare the quantitative assessment of each course with the subjective ones done by students and instructors separately.

g) The department is interested in identifying the potential and prospective employers of its graduates, based on the data from five years. For this, it intends to compare the students strengths per each elective course offered in last five years with alumni survey conducted each year (for last five years) and the input from departmental industrial advisory board.

h) In order to improve academic standards, the department wishes to develop repository of periodic data related to program assessment that includes coursework, exams, analysis of learning outcomes and objectives, etc.

The scenarios ' $a$ - $h$ ' are typically related to academic administration of an engineering department to develop student profiles, though it can be tuned for any other department in an academic institution. Generally, the student information in existing information systems (management or educational) is available in multiple systems and typically distributed throughout the environment; however providing the right information at the right time to the right user has remained a serious problem and input from an intelligent and experienced user is always required to gather the required information. An intelligent system would require integration of interrelated data from different sub-systems in order to produce meaningful results for queries such as $a-h$. With the help of semantic web framework, it can be argued that the role of an intelligent user can be supported to minimize the time to gather all required information.

\section{The SEMANTIC Web FrameWORK}

In order to enrich underlying data layer with well defined meaning, a machine processable semantics layer can be provided using semantic web technology. In this section, techniques used in Protégé [16] are used to simulate various semantic web management activities such as ontology management, semantic annotation and semantic query of annotation triples. The ontology management, semantic annotation and semantic query of annotation triples are designed and exemplified to demonstrate the potential usefulness of semantic web technologies in supporting academic administration environment.

It can be argued that such queries can be handled by simple database searches, if well defined schemas are used. However such queries will become extremely complex due to the main reason that such databases of different management systems are not designed at the planning level to define relationships between their data, hence data traversing gets complicated. Due to this fact, a set of ontologies is developed to contextualize the related data to generate semantic web framework.

\section{A. Ontology Map and Specification}

Ontologies have emerged as explicit formal specifications of the terms in the domain and relations between them [17]. They have become common on World Wide Web and range from large taxonomies categorizing web sites to categorizations of type of relations between different objects on a same server. Many disciplines now develop standardized ontologies that domain experts can use to share and annotate information in their fields such as medicine, chemistry, biotechnology, social sciences, etc. Ontology defines a common vocabulary for web users who need to share information in a domain. It includes machine-interpretable definitions of basic concepts in the domain and relations among them. Some of the general uses of ontologies are to share common understanding of the structure of information among people or software agents, to enable reuse of domain knowledge, to make domain assumptions explicit, to separate domain knowledge from the operational knowledge and to analyze domain knowledge.

Different ontologies developed for different scenarios may be combined to tackle somewhat relating issues. These are related to each other by mapping the output of one query based on a particular ontology to another query based on some other ontology. This type of integration is getting popular in commercial systems as well. For example, a controller mechanism based on various educational ontologies is suggested in [18] to connect knowledge framework with BlackBoard@ Systems.

In order to formally develop ontology for typical scenarios, say for example $a-h$ in section 3, a conceptual map is drawn first by connecting various entities in the respective environment. For a typical university college, like UAE University- engineering department (UAEU-E), this is shown in Figure 1. The Figure 1 shows higher level relations between entities in a conceptual way. The relations are further defined to develop exact associations between the relations of these entities. For example, the entity 'faculty' is related with entity 'electives' through the relation 'expertise'. This relation "expertise” is further defined in different type of expertise such as domain knowledge, industrial experience, research experience in the area, etc. 


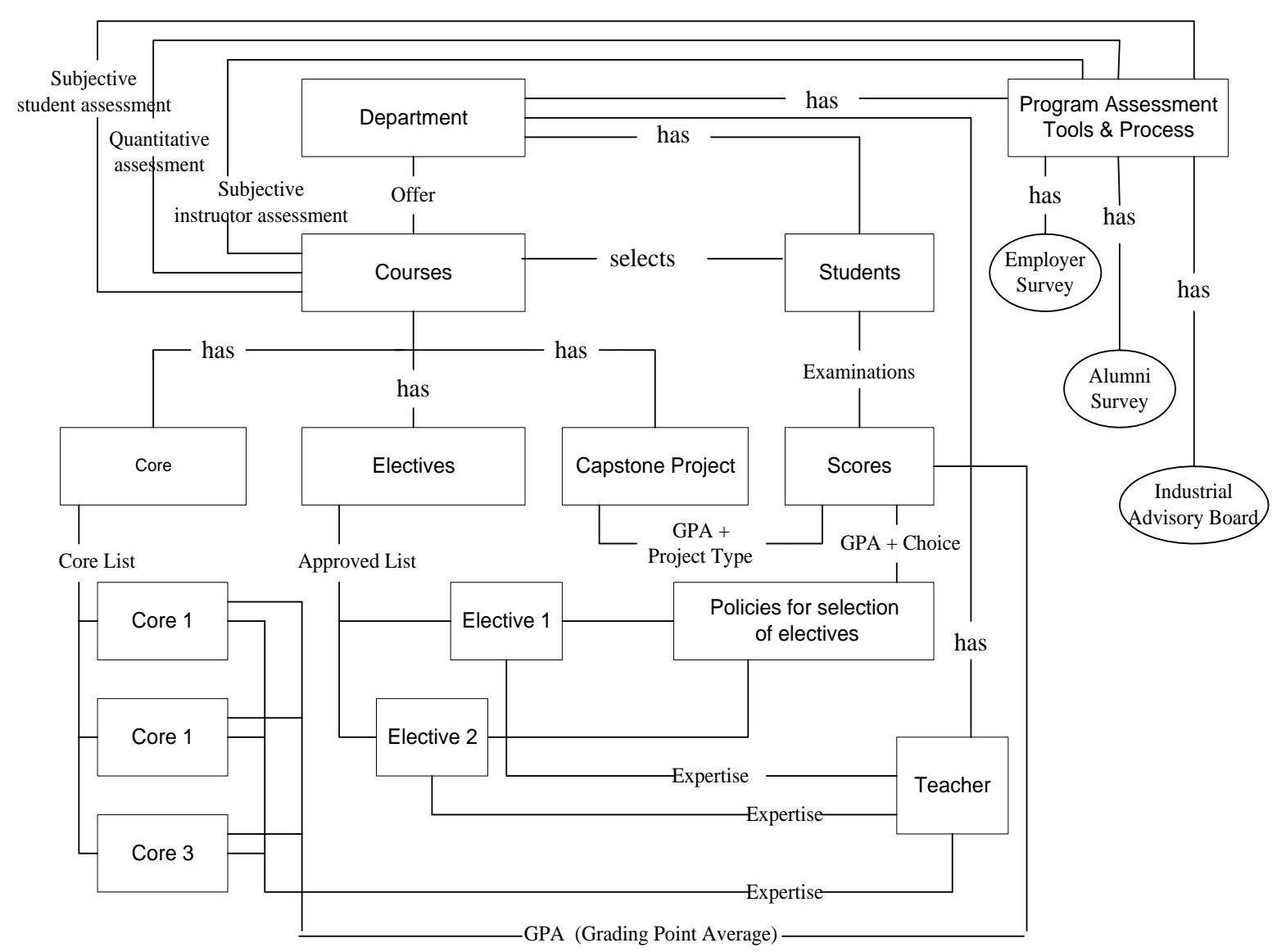

Figure 1. A Conceptual map showing (engineering) entity relationships

The web ontology language (OWL) is a family of knowledge representation languages for authoring ontologies, and is endorsed by the World Wide Web Consortium. This can be used to define exact associations between the relations of the entities. Using OWL for this purpose, the names of lead players are identified in the environment, as shown in Figure 2. The Figure 2 depicts ontology development for the conceptual map shown in Figure 1. It shows how annotations are programmed into Protégé. The highlighting on course specifications is there for the reason that the Protégé environment forces that at least one annotation is selected at any time. The corresponding OWL code is also highlighted. This figure also shows the type of programs and the corresponding outcomes.

Next, the mapped entities are entered into Protégé to develop ontology specification. As an example, the Figure 3 defines conceptual map to develop student profiles by elaborating different relations between entities used in the framework. This specification can be combined with a graph data format tool (like Resource Description Framework document (RDF)) to create a unified database of information, which will contain information of different students' profiles. The RDF is a well known directed, labelled graph data format for representing information on the Web.

Competencies related to students' profiles may be generated as outputs by populating the data over these specifications. For example, student grades of particular subjects are to be collected from exams database. Similarly, student's participation in particular curricular or co- curricular activities carries weight for 'skills development'. Student's completed courses' learning outcomes also help in developing competency of a student over a particular skill. Once ontologies are developed, the framework is a set of such ontologies working together to run intelligent queries. This is exemplified further in section 4.4 where reuse of semantic annotations is discussed.

\section{B. Semantic Annotation}

The semantic annotations are defined as metadata for a particular entity and its relations. They are also used to define the process generation of such metadata. After ontologies are developed for an environment, the end users are envisaged as using the ontologies to annotate resources in the scenarios. The idea is to filter and mould the data at the input level as per our designed ontology. Annotation of resources may also be carried out using RDF, based on certain ontologies. Additional semantics could improve the search power. Adding semantics to end results (in our case generated web pages) means that it is possible to make these distinctions explicit in the content itself and search systems can ignore homonyms but find synonyms.

To demonstrate semantic annotation, some queries are simulated in Protégé, as shown in Figure 4. For this, UAE-E ontologies are loaded in Protégé to allow annotating student information available in the engineering department. An ontology driven template-based instance generation method is used in Protégé to allow semantic annotation through matching instances with ontology definitions. 


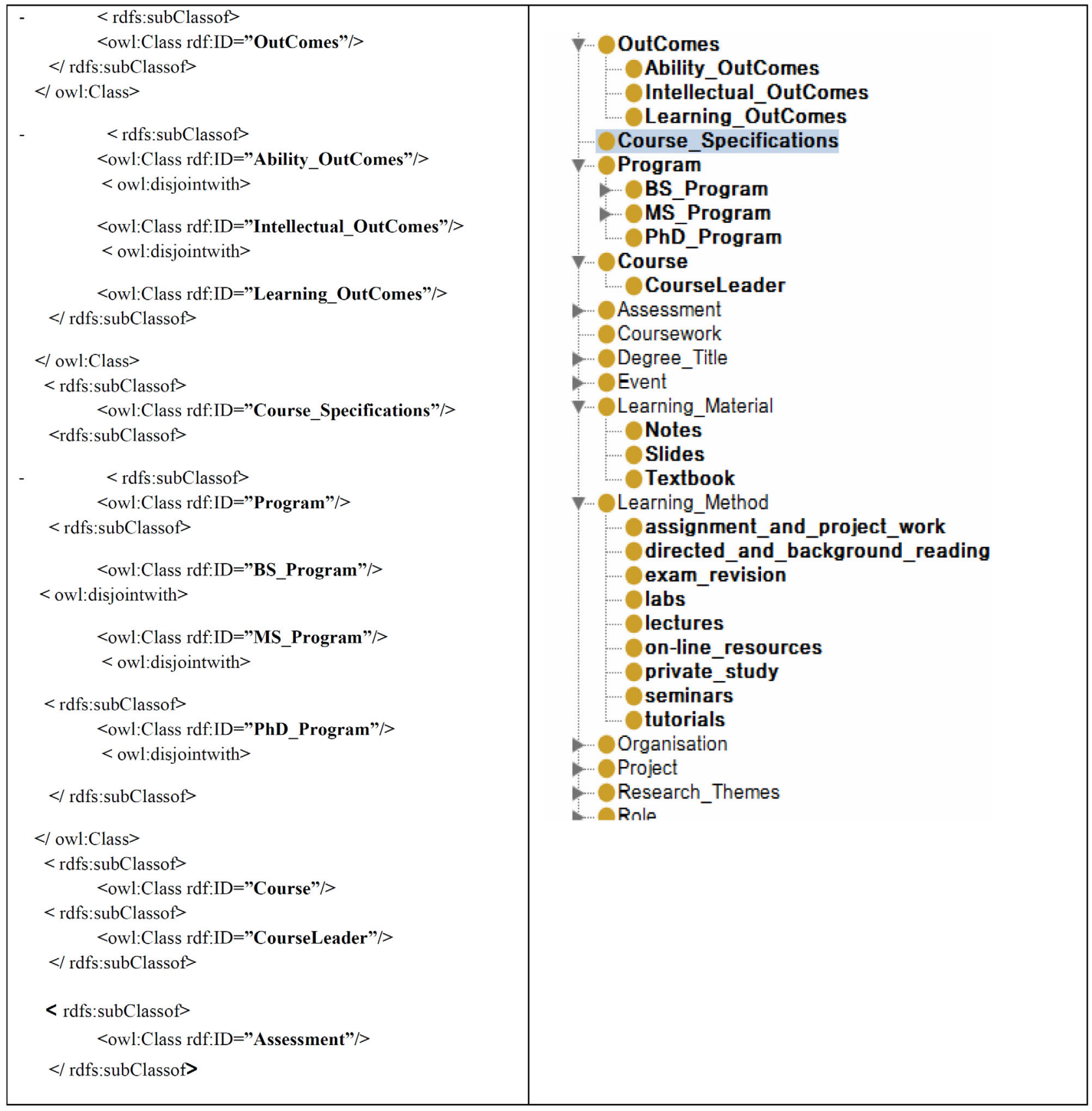

Figure 2. Ontology Development using OWL

\section{Generating Semantic Annotations}

University programs and corresponding courses are always tailored to a common goal. Courses that are taught in a particular program are designed in a way that their outcomes are cumulated for the academic program's common goal. This forms a natural hierarchy between program objectives and course outcomes. Similarly the co-curricular and extra-curricular activities carried out by students also comply with the main goals of the program that the student is progressing through. The idea is to map that hierarchy and create a conceptual map that can be specified using semantic technologies.

The program objectives form the key basis to develop ontologies. The program objectives and outcomes form the basis of rules that are to be matched through our defined ontologies. Similarly these objectives and outcomes benchmark the criteria that are to be achieved by querying student records. In other words, the main context of this work is to map student achievements and scores as per University's defined objectives and outcomes set for a particular academic program.

For example, the program objectives and course outcomes for a typical electrical engineering course (such as Digital Image Processing) are defined as follows:

\section{1) Program Objectives}

- Provide graduates with a high level of analytical and applied skills necessary to actively participate in technology innovations in addition to maintaining the present ones in the country and abroad. 




Figure 3. Conceptual Map to develop student profiles

- Promote the interaction between the university and the local industry. The industry is encouraged not only to actually participate in selecting the various courses and their contents but also have an effective role in endorsing the research themes of the students especially those on study leave from the industry. Consequently, co-supervision from qualified scientists and researchers from the industry is encouraged.

- Promote the creative thinking skills among graduates necessary for life-long learning.

- Promote scientific research and development (R\&D) activities.

\section{2) Course Ability Outcomes}

- To compare and use different tools for image analysis in a transformed domain (wavelet vs. Fourier transform)

- To implement with Matlab image processing algorithms aimed at compression, segmentation, representation, description, and object recognition
- To apply the notions learned in the course to practical image processing problems.

In order to demonstrate this, the student profile rating is used where courses' instances are created under uaeengg:program-ware object, which are later exported through Protégé to RDF. RDF is used as a method for conceptual description or modelling of information that is implemented in uae-engg:program-ware object. It is based upon the idea of making statements about resources in the form of subject-predicate-object expressions. These expressions are known as triples. The subject denotes the resource, and the predicate denotes traits or aspects of the resource and expresses a relationship between the subject and the object. The semantic annotations refer to the RDF triple statements using instance Uniform Resource Identifier (URI) and ontology property as their subject and predicate respectively, e.g., <MSc EE, uaeu-ee:MS Program,Electrical Engineering $>$ and <ELEC 616, uaeuee:course_title, Digital Image Processing $>$. 




Figure 4. Ontology Development in Protégé environment

The following sample code shows how the objectives and outcomes are defined in RDF. In this example the code shows the general objectives for M.Sc. program in Electrical Engineering as: developing analytical skills for handling technology, developing interactive skills for factory environment, creating thinking skills for lifelong learning and creating research and development skills, whereas learning outcomes for the course ELEC 616 (Digital Image Processing) are: comparison of image processing tools, implementation of Matlab image processing algorithms and handling real world image processing problems.

<uaeu-engg:Program rdf:ID="MSCEE">

$<$ uaue-engg:hasobjective="\#1analytical skills for handling technology"l>

<uaue-engg:hasobjective="\#2interative skills for factory environment: local and global"/>

<uaue-engg:hasobjective="\#3creative thinking skills for life-long learning"/>

<uaue-engg:hasobjective="\#4research and development skills"/> <uaeu-engg:hascourse rdf:resource="ELEC 616"/>

<uaeu-engg:course title rdf:resource="\#Digital Image Processing"/> $<$ uaeu-engg:courseoutcome:resource="\#1 comparison of image processing tools"/>

<uaeu-engg:courseoutcome:resource="\#1 implement Matlab image processing algorithms"/>

<uaeu-engg:courseoutcome:resource="\#1 handle real world image processing problems"/>

$<$ /uaeu-engg:Program>

The Figure 3 forms the basis of ontology used to record the possible outcomes coming through various stages of interaction of student related operation. At each node data is generated which is used to generate students' profile. This profile is later used for querying different predefined scenarios. Once instances are created for student related operations, as per the relations defined in Figure 3, these are then used for classification of courses; defining relation between the course and the course teacher and are re-used to calculate teaching load; listing course outlines which will be used to create student's profile; and course assessment mechanisms which are used to develop assessment strategies. Similarly all related instances are to be re-used to extract intelligent information.

\section{Reuse of the Semantic Annotations}

The semantic query of annotated triples is exemplified in this section to understand use of semantic web framework. The Simple Protocol and RDF Query Language (SPARQL) [19] is now considered as the main RDF querying language for semantic web. The specification defines the syntax and semantics of the SPARQL query language for RDF. SPARQL can be used to express queries across diverse data sources, whether the data is stored natively as RDF or viewed as RDF via middleware. SPARQL contains capabilities for querying required and optional graph patterns along with their conjunctions and disjunctions. The results of SPARQL queries can be results sets or RDF graphs.

SPARQL is a query language designed for querying semantic web triples. For this purpose, a part implementation of the scenario is used where an employer wishes to search profiles of graduating students for specific skills and abilities. For example, assume that the employer is looking for students with good skills in signal processing, adaptive filters, image acquisition, sampling, quantization, along with some communication and presentation skills. Developing semantic relations between outcomes 
of student union/sports, co-curricular activities, with the outcomes of academic programs and course intellectual and ability outcomes, it becomes possible to entertain such high level query. Assuming that the above entities have been sufficiently annotated using a shared ontology, such sort of match-making can be carried out through carefully designed semantic web queries into these semantic annotations in the form of RDF triples. By sufficient annotations, it means the minimum required metadata that can handle queries of predefined scenarios. For example queries are made for employers who are looking for students with good knowledge in some particular subjects along with soft skills of communication and comprehension. For this type of query, scores of the subjects are needed that employers are looking for along with student records of certain co-curricular activities where soft skills are also recorded and made part of the student profile.

Ontologies are rather like classification schemes. They are ways of defining the relationships between objects. Ontologies are also used as a classification scheme, which is usually a way of organizing objects by placing them under subject categories. The Figure 5 shows a logical layered architecture of the semantic web [20] followed for academic program administration. In the Figure, RDF Schema (RDFS) extends RDF vocabulary to allow describing taxonomies of classes and properties, where as Extensible Markup Language (XML) layer with XML namespaces makes sure that there is a common syntax used in the semantic web. It can be easily seen from the Figure 5 that the architecture is an illustration of hierarchy of web languages and technologies, where each layer exploits and uses capabilities of the layers below. It can be seen that SPARQL and Ontologies, both are exploiting the capabilities of RDF structure of the application. Ontologies are used to define semantic rules for the information organization in the application, whereas SPARQL is used to query these data sources.

The most straight-forward solution to realize the scenario is to carry out a set of SPARQL queries and postprocessing operations. The following steps are used:

a) Query regarding student profile to check his area of expertise (e.g. in which subjects he has taken good scores).

b) Ordering of the outcomes as per accolades (for example if the student has achieved grade $\mathrm{A}$ in the course of Signal Processing, outcomes of the course will be ordered at higher position).

c) Query regarding student profile to check his cocurricular and sports activities (e.g. in which activity or sport he has won a prize).

d) Query regarding student profile to check his cocurricular and sports activities (e.g. in which activity or sport he has participated).

e) Measuring the distance of the student profile to the requirements of the employer.

f) Rank the candidate's position by the distance.

Below are some examples of the SPARQL queries to retrieve semantic description of outcomes of the courses and the outcomes of the university's sports and cocurricular activities. By referencing to the predefined ontology, it is possible that any other parties recognizing the ontology can understand and reuse these semantics easily.

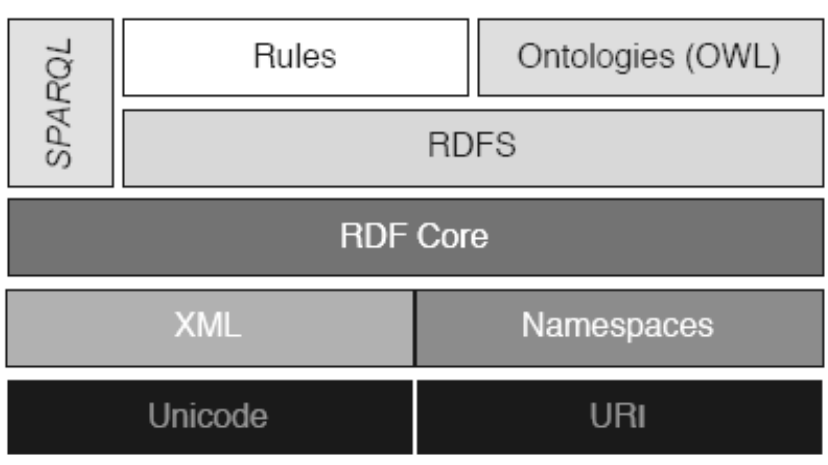

Figure 5. Layered architecture of Semantic Web Application

PREFIX uaeu-engg_ins:

$<$ http://www.uaeu-ee.ae /uaeu-engg_ins.owl\#>

PREFIX uaeu-ee: <http://www.uaeu-ee.ecs.ae/ontology/uaeu-ee.owl\#> SELECT ?s ?p ?0

WHERE $\{$ uaeu-engg_ins: student? courses? scored A $\}$

ORDER BY ?courses

1 ( $p p=$ uaeu-engg:firstName )

( ?o ="f_name"^^<http://www.w3.org/2001/XMLSchema\#string>)

2 ( $? \mathrm{p}=$ uaeu-ee:lastName )

(?0="I_name" ${ }^{\prime \wedge}<$ http://www.w3.org/2001/XMLSchema\#string>)

3 (?p = uaeu-ee:assessment-grade) ( $? 0=$ uaeu-engg_ins:ELEC 615)

4 (?p = uaeu-ee: assessment-grade) (?o = uaeu-engg_ins:ELEC 616)

5 ( ?p = uaeu-ee:assessment-grade-level)

( ?० "A"^^<http://www.w3.org/2001/XMLSchema\#int> )

The above query (select the courses where student $x$ scored A in previous subjects) will yield the result: "scored A in ELEC 615 Adaptive Signal Processing, and ELEC 616 Digital Image Processing”. In the next query, the outcomes of courses are exported:

SELECT ?s ?p ?0

WHERE \{uaeu-engg_ins: ELEC04 ?p ?0

ORDER BY ?p

1 (?p = uaeu-engg:has Learning Outcomes) (?0 = uaeuee_ins:

ELEC615 Learning Outcomes)

2 (?p = uaeu-engg:has_objective) (?o = uaeu-engg_ins: applications of adaptive signal processing)

3 ( $? \mathrm{p}=$ uaeu-ee:has objective) ( $? 0=$ uaeu-engg ins: adaptive filters)

4 ( $? p=$ uaeu-ee:has_objective) ( $? 0=$ uaeu-engg_ins: beam formers)

5 (?p = uaeu-engg:has_objective) (?0 = uaeu-ee_ins: optimum space/time processors)

$6(? p=<$ ttp://www.w3.org/1999/02/22-rdf-syntax-ns\#type>) $(? 0=$ uaeu-engg:Course)

The above queries will return a list of matching results of students with the courses in the areas of signal and image processing, and their respective learning outcomes. In the same way, the student's participation in university's club/sport activities and college's co-curricular activities may be queried. A sample query may be shown as:

SELECT ?s ?p ?0

WHERE $\{$ uaeu-engg_ins: ?s ?p ?o $\}$

ORDER BY ?p

1 (?p = uaeu-engg:has College Activities ) (?o = uaeu-ee_ins: Debate Competition)

2 (? $p=$ uaeu-engg:has_First_Position ) ( ?0 = uaeu-engg_ins: student $\mathrm{x})$

$3(? p=$ uaeu-ee:has_Second_Position ) ( ?o = uaeu-engg_ins: student $y)$

4 (?p = uaeu-ee:has_Third_Position ) ( ?o = uaeu-engg_ins: student z) 5 (?p = <http://www.w3.org/1999/02/22-rdf-syntax-ns\#type>) (?o = uaeu-engg:College) 
SPARQL queries for the said purpose are developed manually, however tools are available that provide simple and user friendly interface to develop queries which allow to select different entities, their relations, variables defined, and associated parameters. The next step is to join the results of these queries for student $x$, which can be done by a simple join command. Then they should be sorted by results in subjects and positions in co-curricular and sport activities. This will create an ordered list, sorted as per the measure of the distance of student's achievements to the employer's requirement. In this way, complex queries can be jointed to develop student's profile and finally matched with the employer's requirement. Data in the traditional repository is then opened up through a set of semantic activities.

\section{Discussions}

Like any semantic web application, challenges include manageable set of concepts and assertions, vastness, vagueness and inconsistency. Automated reasoning system will have to deal with these issues in order to deliver onto the promise of semantic web. Cost of adding new concepts and assertions is another challenge that we have to deal with. It will become tediously difficult to map new concepts and assertions along with the old one. Uncertainty is another challenge that Semantic Web applications face. For example, a student portfolio might present a set of outcomes which correspond to a number of different distinct areas of expertise, each with a different probability. Probabilistic reasoning techniques are generally employed to address uncertainty in such circumstances, which the authors feel are beyond the scope of this paper.

Semantic technologies also claim of enhancing intelligence and provide meaningful information for searching and browsing. Ontology consensus has appeared to be a major issue in implementing semantic technologies. This is mostly due to different implementation procedures cross departments or cross organizations. Ontologies typically describe more structure than dictionaries [21], e.g., in their arrangement of concepts into an is-a hierarchy (that is, into a taxonomy). They may have more precise semantics than taxonomies, e.g., by identifying attributes associated with a given concept, and possibly rules governing the values that the attributes assume.

There are two approaches to implementation of the semantic web. In the bottom-up approach, all known components and interactions are integrated to model the system, where as in top-down, the system is taken first and then decomposed it into component parts and interactions. The critical difference surfaces clearly when knowledge of all of its components and interactions is not known. Example implementations exist for either of these approaches, for example in case of bottom up approach the semantic annotations (meta-data) are embedded right into the data. The opposite camp is exploring the topdown approach, which relies on analyzing existing information. In that, a developer models the semantics of a vertical domain (such as, farm equipment, movie cameras, etc.) and the kinds of things users would want to do in that domain, and then links web pages into a meaningful series of information views and user actions. This approach seems natural to a grown up university to employ semantic web to existing sub-systems to exploit diversity of data to its advantage. The ultimate top-down solution would be a fully blown natural language processor, which is able to understand text like people do.

The semantic web can be compared with semistructured data model. In semi-structured model, there is no separation between the data and the schema, and the amount of structure used depends on the purpose. Some of the advantages of this model include flexible format for data exchange between different databases, viewing structured data as semi-structured (for browsing purposes); that the schema can easily be changed; and portable data transfer format. The primary trade-off in using a semi-structured database model is that queries cannot be made as efficiently as in a more constrained structure, such as in the relational model. For unstructured or semistructured data, a relational database management system (RDBMS) has greater difficulty, and query performance is usually unacceptable for relatively large amount of data. Typically the records in a semi-structured database are stored with unique IDs that are referenced with pointers to their location on disk. So for doing searches over many records, it is not as efficient because it has to seek around the disk following pointers. For semi-structured databases, despite the simple syntax of the constraints, their associated implication problem is R.E. complete and finite implication problem is co-R.E complete [22].

As the semantic web is based on semantically defined ontologies of inter-related and interconnected datasets, it is free of the query based on the user location or the type. As long as a query processing system understands the schema and semantics of the database, it will be able to return informative response to the user. Thus the semantic framework presented in this work is independent of the regional or cultural issues underlying the need to perform different kinds of searches.

The other point investigated in this concept is whether it is possible to extend the ontology to departments other than engineering. The general opinion is that it remains the same as far as it is applied to an individual department. In order to understand how shall it be perceived if the concept is extended to academic administration of the college with many departments underneath (that is, how does it look to users who control and monitor a college which has many engineering departments), at the institutional level it seems that if this ontology is applied at a higher scale, its modus operandi will remain the same, except that this time, it will be more complex and more data-loaded. As an example, at College of Engineering of UAE University, initially (say first year), courses remain the same, but then student specializes by selecting a department. Still a student can strengthen ones credentials by selecting (some) free electives from any department (during last year of studies) or by going through an interdisciplinary senior (final) year project. In this case, it means that semantic layer shall retrieve data from all departments and project the profile of a student within a college. Generally, it all depends upon the strategies defined by different departments within a college. Ontology is a way to connect these complex or loosely defined rules and concepts. The complexity of the integrated system increases once additional criteria are added to further highlight student's major achievements (like star rating tag comparison and clouding, etc).

The view of this layer can be generalized to stake holders (say college industrial advisory board or any stake holder) of the program through Internet, assuming 
university is willing to provide due access of the system. This concept may also be very useful to the university, i.e., instead of acting on the query of employer, the university should catalogue its students telling the employers that they are 'fit' for $x$ or $y$ type of industries. But for this, a proper categorization within a country-specific industrial sector may be needed, to be mapped against the skills of the students.

\section{CONCLUSIONS}

The effort exercised in this work demonstrates the effective use of semantic web in a typical environment like an engineering college of a university. The implementation was exemplified by developing student profiles as student profiling has become an important aspect of any growing academic system. The demonstrated benefits in this work are reduced cyclic and tiring work, quicker decision making at needed time, etc. The implementation of semantic web approach towards academic program administration helps in program accreditation process, as queries may be set to the system to dig out useful and needed information about student achievements. It was also demonstrated that semantic web approach may be applied to any academic program administration. However, the complexity of semantic web increases once additional criteria or sub-systems are added to the integrated platform. It was discussed that top-down approach seems more natural to integrate existing data through semantic web, though it depends on academic institutions how strategy is defined. In order to improve capacity on this development, web services or web portals can be examined to include more complex functions at service level or for end users.

\section{REFERENCES}

[1] Botev C, Chao H, et. al., Supporting Workflow in a Course Management System, Proceedings of ACM Technical Symposium on Computer Science Education, February 23-27, St. Louis, Missouri, USA, 262 - 266 (2005).

[2] Moule P., Developing the Communities of Practice, Framework for E-learning, Electronic Journal of E-Learning, 4(2), (2006).

[3] Mair R., Knowledge Management Systems: Information and Communication Tech for Knowledge Management, $3^{\text {rd }}$ Edition, Springer Verlog, (2007).

[4] Lopez G., Technological Capabilities Indicators of the State University, New Organizational Tools for its Management Guiding and Strengthening, and Knowledge Building and Transfer, Proceedings of IEEE Engineering Management Conference, Managing Technologically Driven Organizations: The Human Side of Innovation and Change, Albany New York USA, 2-4 Nov. (2003).

[5] UAE University, UAE, http://www.uaeu.ac.ae/

[6] T. Berners-Lee, J. Hender, and O. Lassila, The Semantic Web, Scientific American, 284(5), 34-43 (2001). doi:10.1038/scientific american0501-34

[7] Wikipedia, http://en.wikipedia.org/wiki/Semantic_Web
[8] Herman, Ivan, Semantic Web Activity Statement, W3C: http://www.w3.org/2001/sw/Activity.html. Retrieved 2008-03-13.

[9] Feng (Barry) Tao, Shakeel A. Khoja, Hugh Davis, Andy Gravell, Academic Administration and Management Scenarios on the Semantic Web, Proceedings of the 2008 Eighth IEEE International Conference on Advanced Learning Technologies, Santander, Cantabria, Spain, 149-151 (2008).

[10] Autology Personalized learning, http://autology.org/index.html

[11] MIT Course Picker (beta), http://picker.mit.edu/

[12] Gray, N, Linde, T, and Andrews, K, The SKUA Project and the Semantic Web, Proceedings of Astronomical Data Analysis Software and Systems, Quebec, Canada, November 2-5 (2008).

[13] DePiero, F, NetExam: a Web-based Assessment Tool for ABET2000", Proceedings of $31^{\text {st }}$ Frontiers in Education Conference, 2, October 10-13, Washington, DC. (2001).

[14] EdScence Project, http://www.edscene.ecs.soton.ac.uk/

[15] Imielinski, T, "Intelligent query answering in rule based systems", Journal of Logic Programming, 4(3), 229-257 (1987). doi:10.1016/0743-1066(87)90003-3

[16] The Protégé ontology and knowledge acquisition system http://protege.stanford.edu/

[17] Gruber T R, Toward Principles for the Design of Ontologies used for Knowledge Sharing, International Journal of HumanComputer Studies, 43(5-6), 907-928 (1995). doi:10.1006/ijhc. 1995.1081

[18] Craig McKenzie, Alun Preece, and Peter Gray, A Semantic Web Blackboard System, 4th Workshop on Principles and Practice of Semantic Web Reasoning, Budva, Montenegro, June $10^{\text {th }}-11^{\text {th }}$ (2006). doi:10.1007/11853107_15

[19] SPARQL Query Language for RDF: W3C Recommendation 15 January 2008; http://www.w3.org/TR/rdf-sparql-query/

[20] Aurona Gerber, Alta van der Merwe, Andries Barnard, A Functional Semantic Web Architecture, European Semantic Web Conference, 273-287 (2008).

[21] Bailin, SC, Berg-Cross, G, Finin, T, Panel Discussion on Ontologies, Lecture Notes on Computer Science, 2564, DOI 10.1007/b83557, Springer Berlin / Heidelberg (2003).

[22] Peter B., Wenfei F., Scott W., Path Constraints on Semistructured and Structured Data, Proceedings of the seventeenth ACM SIGACT-SIGMOD-SIGART symposium on Principles of database systems, Seattle, 129-138 (1998).

\section{AUTHORS}

Q. A. Memon is with the Electrical Engineering Department, United Arab Emirates University, Al Ain, 17555, United Arab Emirates. He has published about seventy research papers in International Journals and Conferences (e-mail: qurban.memon@uaeu.ac.ae).

S. A. Khoja is with Faculty of Computer Science, Institute of Business Administration, Karachi, Pakistan. Previously, he was research fellow with Learning Societies Lab, School of Electronics and Computer Science, University of Southampton, UK. He has published over thirty research papers in International Journals and Conferences (e-mail: skhoja@iba.edu.pk).

Submitted August $29^{\text {th }}, 2010$. Published as resubmitted by the authors November $22^{\text {nd }}, 2010$. 